\section{Identification capacities of retarded and normal children*}

\author{
JEROME SMITH and HERBERT KAUFMAN† \\ University of Connecticut, Storrs, Conn. 06268
}

The effects of information load, delay of response, and number and type of dimension of stimulus variation upon the identification capacity of retarded and normal children were examined. A retardate deficit was found, characterized by a generally lower level of information transmission and increasing retardate-normal differences as task demands increased.

In the history of perceptual investigations, those questions dealing with the discriminative capacities of organisms have long held an honored position. Dating from Weber's and Fechner's work in the 19 th century, data concerning difference thresholds have been collected in laboratories all over the world. In contrast to discrimination, an equally fundamental perceptual process, that of identification, has received a great deal of experimental attention only in relatively recent history. Attention was focused on the identification process by Miller's influential article, "The magical number seven, plus or minus two" (Miller, 1956). While the Weber fraction may vary considerably across a group of selected physical dimensions, Miller pointed out that the number of identifications possible within each of these dimensions was fairly invariant. This invariance has been demonstrated for many dimensions in many studies (Fitts \& Posner, 1967).

The experiment to be described was designed to discover whether identification capacity varies with the intellectual and/or developmental level of the $S$. The question seems a reasonable one to ask, especially with respect to possible differences between retarded and normal children. Identification is a process that has not been carefully studied in either retarded or normal children, while other basic processes have received considerably more attention, for example: discrimination learning (Zeaman \& House, 1963), short-term memory (Belmont \& Butterfield, 1969; Scott \& Scott, 1968), long-term

*This research was supported by an Office of Education grant, No. OEG-1-70-0101, to the two authors.

†The authors are indebted to Mr. William Lang, Principal, and the teachers and students of the Hall Memorial and Willington $C$ enter schools for their cooperation. We also wish to thank Drs. David Zeaman and Betty House, in whose laboratory the retarded Ss were run. Data collection was made possible through the able assistance of Donna Hammel Terry Chotniky, Pat Meguire, and Ed Pollack. memory (Belmont, 1966), and sensory acuity (Kodman, 1963).

Identification capacity is studied by increasing the number of stimuli to be identified until errors begin to occur. The changes in identification become extremely interesting when viewed in terms of the information transmission characteristics of the $S$. As the stimulus pool is increased in size (increased stimulus information), the $\mathbf{S}$ transmits information (identifies) perfectly up to a certain point. At that point, he continues to transmit the same amount of information or less, even though the stimulus information (size of the pool) may increase. This asymptotic value of transmitted information is referred to as channel capacity. The invariance of identification across dimensions that Miller pointed out becomes clearer when viewed in terms of channel capacity. We know that channel capacity varies as a function of whether the stimuli to be identified vary unidimensionally or bidimensionally (Eriksen \& Hake, 1955), and as a function of the manner in which dimensions are combined in multidimensionally varying stimuli (Kaufman \& Levy, 1971). We know little about the relation of channel capacity to subject differences. Recently, Olson (1971) has suggested that channel capacity may be related to mental age. Our study examines channel capacity differences in identification in retarded children and their normal mental age equivalents in first grade as well as their normal chronological age equivalents in sixth grade.

The conventional identification situation requires the $S$ to provide a previously acquired verbal label to the stimulus presented. For example, "This is a green square," or "That is number five." The identification procedure we use has been conventionally described as delayed match-to-sample or recognition. We present the stimulus object, remove it, and then offer the entire pool of objects for that experimental condition, enabling the $S$ to point to the one he just saw. We feel that our procedure and the conventional identification procedure are similar in the fundamental sense that the distinctiveness of the original stimulus provides the basis for response. One important difference between the two procedures lies in the necessity for having a well-learned verbal label for the stimulus in the conventional identification situation. In dealing with young children, and retardates in particular, verbal responses may pose a problem. The absence of or difficulty with verbal labels does not necessarily mean that the child cannot recognize the unique characteristics of the stimulus. The use of our procedure is further justified by the fact that it produces regular data when used with such nonverbal Ss as monkeys (Kaufman \& Wilson, 1970).

In addition to the intellectual and developmental level of the Ss, the independent variables of this study include stimulus information load (size of the stimulus pool to be identified), number and types of dimensions along which the stimuli vary, and the delay between removal of the stimulus to be identified and the production of the pointing response. The information load variable provides the key to possible channel capacity differences among our $S$ groups; the number of dimensions variable tells us about possible differential effects of redundancy, and the delay variable may point to normal-retardate memory differences.

\section{SUBJECTS}

Six retarded children, six normal first graders, and six normal sixth graders served as Ss. The retardates, five male and one female, were residents of the Mansfield State Training School, Mansfield, Connecticut. They were mixed diagnostically, including three familial, two mongoloid, and one brain damaged child. None of the children exhibited gross sensory or motor impairment, all attended public school classes at the training school, and all had previously served as $\mathrm{Ss}$ in discrimination learning experiments. Their mental ages ranged from 56 to 75 months (mean $=62$ ), while their CA ranged from 140 to 164 months ( mean $=145$ ). The normals, three boys and three girls at each grade level, were selected by their teachers as "average" students in the Hall Memorial and Willington Center public schools, Willington, Connecticut. The $\mathrm{CA}$ range for the first grades was 73 to 83 months, with a mean of 76 , with a CA range for sixth graders of 142 to 150 and a mean of 147 months.

Since MA levels for the normais were not available, the selection of "average" students was requested of 
the teachers. Average was defined as neither above nor below grade level but doing reasonable work in the opinion of the home room teachers.

The discrepancy in sex distribution between the normals and retardates resulted from the feeling that any possible sex effect would be minimal in comparison to $\mathrm{CA}$ and $\mathrm{MA}$ effects. The retardates were therefore chosen to reflect the best possible matches on the latter two variables and sex balance was sacrificed to this end.

\section{MATERIALS}

The stimuli to be identified were squares that varied in size or brightness or in both size and brightness in different conditions. Size variations were from 2/8-in. to 11/8-in. in 1/8-in. increments. Ten shades of gray selected from a set of 16 Color Aid grays, represented the degrees of brightness variations. Two sets of the 100 possible combinations of size and brightness were constructed by centering each square on a $2 \frac{1 / 4}{4}$-in. white display card and spraying with a clear plastic preservative coating. One of the two sets of cards served as stimuli, while the others were mounted by hinges on a response matrix board.

The response matrix board contained 100 1/4-in.-deep circular depressions, 1 in. in diam, arranged in a 10 by 10 matrix. Each depression could be covered by a hinged display card. The board was mounted almost perpendicular (at an angle of approximately $85 \mathrm{deg}$ ) on a turntable, to permit easy access for both the $\mathrm{E}$ and the $\mathrm{S}$.

\section{PROCEDURE}

On each trial, the $E$ held a stimulus display card up to the $S$ for approximately $2 \mathrm{sec}$, with instructions to look at it carefully. The card was then removed and after the appropriate delay interval, the response matrix was turned toward the $S$, with instructions to point to the card he had just seen. The pool of cards for that particular condition was arranged in natural order on the board (smallest to largest, darkest to lightest), with the empty depressions covered by a white cardboard mask.

The E provided knowledge of results with a verbal "good" or "no." In addition, retarded Ss were permitted to lift the display card on the response board to find $M \& M$ candy in the well when correct. EXPERIMENTAL DESIGN

One between-group variable (retarded, first or sixth graders) and three within-group variables (dimensionality, number of alternatives, and delay) made up the overall experimental design. The levels of the dimensionality variable were unidimensional (size or brightness) and bidimensional (size and brightness combined in a perfectly correlated fashion). The number of alternatives variable had levels of 2,4 , or 6 for unidimensional stimuli and 6 or 8 for bidimensional stimuli. Since better performance was expected in the bidimensional case, we felt that the smaller number of alternatives would not be necessary in the bidimensional case but that a larger number of alternatives might be required to test bidimensional capacity. The delay between the presentation of the stimulus for identification and the response matrix was set at 0,10 , or $20 \mathrm{sec}$.

All Ss were run on all combinations of the three variables, with eight daily sessions of 90 trials each required of each child. A daily session was divided into the three blocks of 30 trials each. Within each block, the delay levels and particular stimuli chosen for indentification were varied randomly, with the restriction that each value appear an equal number of times. The unidimensional conditions were presented on the first 6 days, with 2 days each of two, four, and six alternatives, in that order. The bidimensional conditions were presented on the last 2 days, with the six alternative ones preceding the eight. The size and brightness dimensions were completely counterbalanced across the unidimensional sessions. If a child performed at the $90 \%$ correct level in any block of 30 trials, the daily session was terminated after that block.

Specific values for a given set of stimuli were chosen from the complete pool or 100 stimuli to maximize the separations among the set and, as was previously stated, were assigned randomly to a given trial in a daily session.

The response measure used was amount of information transmitted (T) in each experimental condition. Transmitted information is calculated from a stimulus-response matrix. The number of responses made to a given stimulus is recorded in each cell with the column totals providing the frequency with which each stimulus was presented and the row totals the frequency with which each response was made. Information transmitted is given by the sum of the stimulus information and the response information minus the cell information. The transmitted information indicates the degree of overlap of stimulus and response information. When the number of response categories is the same as the number of stimulus categories and the $S$ is responding on a purely random basis, $T=0$. If the $S$ is performing perfectly, stimulus and response information will be equal and $\mathrm{T}$ will equal the stimulus information. RESULTS

Because the amounts of stimulus information (number of alternatives) differed in the unidimensional and bidimensional conditions $(2,4$, and 6 alternatives for size or brightness, 6 and 8 alternatives for size and brightness combined), three analyses of variance were performed. The first included unidimensional data only, the second bidimensional data only, and the third compared unidimensional and bidimensional performance at the point of maximum stimulus information ( 6 alternatives for the unidimensional case and 8 for the bidimensional).

\section{Sex}

The performance of males and females was very similar in all phases of experimentation. The overall Fs for sex in first and sixth graders were not significant and there were no indications of any interactions.

Stimulus Information

The effects upon the three $S$ populations of increasing stimulus information and type and number of dimensions available for identification are presented in Fig. 1. The figure illustrates the expected increase in transmitted information with increasing stimulus information, an increase statistically substantiated by the two appropriate variance analyses. The main effect for number of alternatives in the unidimensional analysis was reliable $(\mathrm{F}=16.01, \mathrm{df}=$ $1,255, p<.001$ ), as was the case in the bidimensional analysis $(F=17.08$, $\mathrm{df}=1,75, \mathrm{p}<.001)$. More interesting were the significant differences in information transmitted between the retardates on the one hand and the first and sixth graders on the other (unidimensional $\mathrm{F}=67.47$, $\mathrm{df}=1,15$, $\mathrm{p}<.001$; bidimensional $\mathrm{F}=37.1$, df $=$ $1,15, \mathrm{p}<.001)$.

Although it is clear from Fig. 1 that the retardates were transmitting some information at each stimulus information load, the gain in information transmitted with increasing loads is clearly much smaller than the gain for normals. A test of the gain for retardates alone, however, does produce an $\mathrm{F}$ of 6.76 with $\mathrm{df}=$ 1,25 and $p<.05$. The discrepancy between the gain for retardates on the one hand and normals on the other is emphasized by a significant interaction of intellectual level and number of alternatives $(F=7.04, \mathrm{df}=1,255$, $p<.01)$ in the unidimensional analysis.

While the intellectual parameter clearly affected the amount of information transmitted, the effects of the developmental parameter were not so evident. Although sixth graders 
6th grade

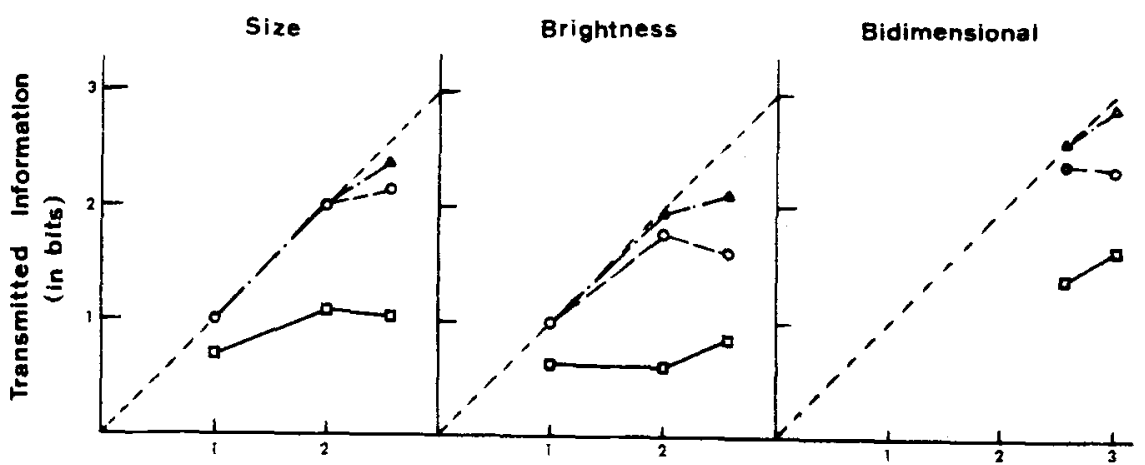

Stimulus Information (in bits)

Fig. 1. Information transmitted by the three experimental groups as a function of the amount of stimulus information (number of alternatives) and type of stimulus variation.

always performed at a somewhat higher level than did first graders, the difference reached statistical significance only in the analyses comparing uni- and bidimensional conditions at the point of maximum stimulus information $(\mathrm{F}=5.52, \mathrm{df}=$ $1,15, \mathrm{p}<.05$ )

Dimensionality

Figure 1 illustrates the fact that performance was superior for all groups when two dimensions were available for identification than when only one dimension was available. This fact was statistically substantiated in the uni- and bidimensional analysis ( $F$ $=228.9$, df $=1,120, p<.001)$. This analysis also revealed the effects of the intellectual parameter, with normals outperforming retardates $(F=44.66$, $\mathrm{df}=1,15, \mathrm{p}<.001$ ) overall. In this analysis, again, an interaction involving intellectual level was found. The interaction was between the normal-retardate comparison on the one hand and dimensionality on the other $(F=4.10$, df $=1,120, p<.05)$. In the unidimensional analysis, increasing stimulus information load (greater demand upon the $S$ ) produced increasing retardate-normal differences. In this analysis, the two-dimensional case (a lesser demand upon the $S$ ) produced a smaller retardate-normal difference than did the one-dimensional case. However, it should be pointed out that the closeness of the two normal groups to the informational ceiling in the bidimensional condition makes the smaller difference. difficult to interpret.

Another type of dimensionality effect is illustrated by the unidimensional analysis. Size proved lst grade $\circ-\infty$

Retardates to be an easier dimension than did brightness $(\mathrm{F}=2.47$, df $=1,255$, $\mathrm{p}<.05$ ) for all groups. Delay

Figure 2 illustrates the delay effects for the six alternative unidimensional conditions and the six and eight alternative bidimensional conditions. The effects of delay between stimulus presentation and response matrix presentation seen in the figure was found with smaller information loads as well (two and four alternatives in the unidimensional situation). All three variance analyses revealed reliable delay effects (unidimensional $F=7.86, \mathrm{df}=1,255, \quad \mathrm{p}<.01 ;$ bidimensional $\mathrm{F}=23.58, \mathrm{df}=1,75$, $\mathrm{p}<.001$; maximum information load $F=26.17, \mathrm{df}=1,120, \mathrm{p}<.001)$. Not only did increasing delays result in decreasing information transmission overall, but they did so differentially with respect to intellectual level. Retardate-normal differences increased as delay increased, a fact statistically substantiated by reliable interactions in all three variance analyses (unidimensional $\mathrm{F}=7.04$, $\mathrm{df}=1,255$, $\mathrm{p}<.01$; bidimensional $\mathrm{F}=8.71, \mathrm{df}=$ $1,75, \mathrm{p}<.005$; maximal information load $\mathrm{F}=4.62$, df $=1,120, \mathrm{p}<.05$ ).

Although there is a greater effect of delay upon retardates, the normal children are not immune to the effects of delay. While the delay functions for first and sixth graders in Fig. 2 do not show marked decrease, an overall test of delay for normals alone did reveal the decrement to be a significant one $(F=4.73, \mathrm{df}=2,110, \mathrm{p}<.025)$.
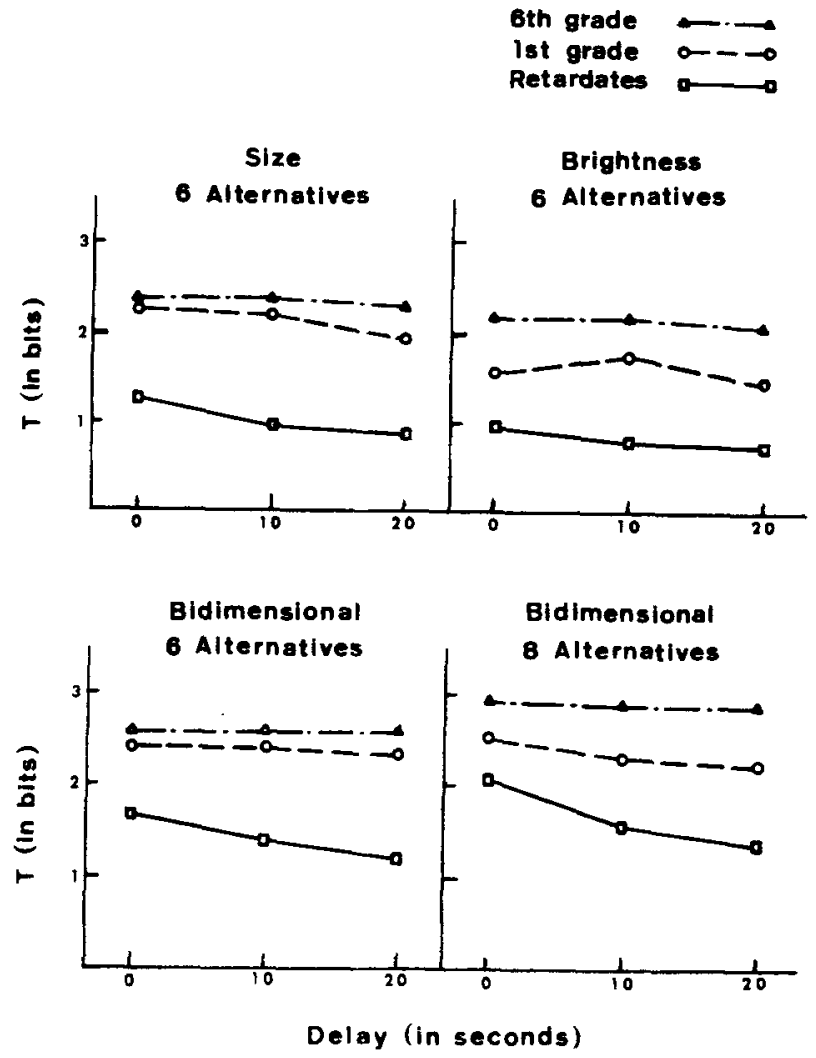

Fig. 2. Information transmitted $(\mathrm{T})$ by the three experimental groups as a function of delay between the stimulus and the response matrix and type of stimulus variation. 


\section{DISCUSSION}

The data of our experiment clearly support the inference that intellectual level plays a role in the ability to transmit information in the identification situation as we have defined it. In all comparisons made, retarded children performed at a significantly lower level than did both their MA and CA controls. This retardate deficit is further emphasized by the interactions of intellectual level with increasing information load (number of alternatives) and with increasing delays between presentation of the stimulus and presentation of the response matrix. In general, as greater demands are put upon the $S$, the retardate deficit increases.

The effect of a developmental parameter of identification is not as clear as the intellectual parameter effect. While sixth graders consistently perform at a level equal to or higher than that of first graders, the differences are small and reach a level of statistical significance only in comparisons involving maximum stimulus information in the uni- and bidimensional conditions. It is possible that a developmental factor is operating, but that it requires a higher information load to reveal its effect. A parametric study including closer examination of the developmental factor would appear warranted.

Given the retardate deficit in identification, the question of the nature of that deficit remains. Our original conjecture was that Miller's magical number in terms of channel capacity (Miller, 1956) might be lower for retardates than it is for normals. That is, with unidimensional stimulus sets on all (or at least many) perceptual dimensions, retardates should reach asymptotic information transmission at a lower level than do normals. Such capacity difference should include not only a lower retardate asymptote but equal retardate-normal ability to transmit smaller amounts of information before asymptote is reached. Our data do not point unambiguously to such a capacity difference. Figure 1 reveals a retardate deficit even when stimulus information is only one bit. Furthermore, as stimulus information increases, retardates do transmit more information but still far below normal performance. It is possible that the notion of a channel capacity deficit is inappropriate. However, our data do not force rejection of a capacity difference but may point to the contribution of other factors as well. One such factor is the context effect, the effect of preceding trials upon an identification response. An analysis of such context effects in the performance by monkeys of a task similar to ours has been outlined by Kaufman \& Wilson (1970). This analysis identifies three types of error, based upon the trial immediately preceding the reference trial:

(1) An $R$ error that entails repeating the response made in the preceding trial when the stimulus has been changed and the previous response was incorrect. This type of error may be thought of as response perseveration independent of the stimulus.

(2) An S error, which is making the response appropriate for the previous trial stimulus when the previous trial response was incorrect. This error may be thought of as a correction of the preceding response.

(3) An S-R error, which is making the response that was correct for the previous trial. This type of error may be thought of as response perseveration dependent upon the preceding stimulus. Errors other than the three described are considered errors of identification independent of context.

The analysis of context effects in our experiment is contaminated by the fact that intervals between stimulus presentations were not equal, since delays were randomly assigned within blocks of trials. However, mindful of the delay problem, we did analyze errors to find that the most common context effect was the incorrect response perseveration ( $R$ error), with the correct response perseveration (S-R) error made less frequently. Very few corrections (or $S$ ) errors were made by our Ss. However, more $S, R$, and S-R errors were made by retardates than by normals, indicating greater susceptibility to context effects as defined by the preceding trial.

Our study has produced what we feel are convincing data pointing to a retardate identification deficit. It seems clear that this deficit is to some extent influenced by the tendency in our retarded $\mathrm{Ss}$ to be more greatly influenced by the preceding trial than are normals. Whether the effects of preceding trials upon retardates result from the tendency to revert to such strategies when faced with a difficult task or whether they enter all tasks with such strategies is not determinable from our data.

It is not clear that the form of the identification deficit may be described in terms of channel capacity differences. The asymptotic levels of information transmission required for such a description are not readily seen in our data. It is possible to speculate about the mechanism underlying the deficit. It might be suggested that the difference between our retarded and normal children represents a simple discrepancy in the ability to attach verbal labels to stimuli. However, our matching task was chosen not only because such verbalizations were not an integral part of the process but because they were unnecessary, as evidenced by the success of such nonverbal $S$ s as monkeys in comparable situations. The effects of delay upon matching also argue against a purely verbal mechanism. It does not seem to be the case that normals mediate their choice by a verbal label, rehearse it, and then make the match as well after longer delays as they would after shorter delays. The normals were subject to delay decrements even though they were not as large as the retardate decrements. If one were to appeal to a verbal mediator, one would have to postulate not only differential effects of unfilled rehearsal periods in normals and retardates, but also a rehearsal decrement over time in normals. What does appear to be true is that the trial-by-trial strategies for retardates differ from those of normals. Response perseveration and correction errors are more characteristic of the retarded group than of either the first or sixth graders. These context effects would tend to depress performance over all levels of stimulus information. They would also tend to obscure channel capacity difference, i.e. differential asymptotic levels of information transmission. Rather than prematurely postulating a mechanism for a retardate identification deficit, we are directing our present research efforts to a clearer specification of the deficit in terms of the possibility of channel capacity differences and a fuller understanding of context effects.

\section{REFERENCES}

BELMONT, J. M, Long term memory in mental retardation. In N. R. Ellis (Ed.), International review of research in mental retardation. Vol. 1. New York: Academic Press, 1966.

BELMONT, J. M., \& BUTTERFIELD, E. C. The relations of short-term memory to development and intelligence. In $L$. $P$. Lipsitt and $\mathrm{H}$. W. Reese (Eds.), Advances in child development and behavior. Vol. 4. New York: Academic Press, 1969. ERIKSON, L. W., \& HAKE, H. W. Multi-dimensional stimulus differences and accuracy of discrimination. Journal of Experimental Psychology, 1955, 50, 153-160.

FITTS, P. M., \& POSNER, M. I. Human performance. Belmont, Calif: Brooks/Cole, 1967

KAUFMAN, H., \& LEVY, R. M. Analysis of the combination of perceptual dimensions. Psychological Bulletin, 1971, 75, 369-378

KA UFMA N, H., \& WILSON , M. Information processing in the monkey. Paper presented at the annual meeting of the Psychonomic Society, San Antonio, October 1970.

KODMAN, F., JR. Sensory processes and mental deficiency. In $N$. R. Ellis (Ed.) Handbook of mental deficiency. New 
York: McGraw-Hill, 1963.

MILLER, G. The magical number seven,

plus or minus two. Psychology Review, $1956,63,81-97$

OL SON, D. R. Information-processing limitations of mentally retarded children.
American Journal of Mental Deficiency, $1971,75,478-486$.

SCOTT, K. G. \& SCOTT, $M$. S. Research and theory in short-term memory. In $\mathrm{N}$ R. Ellis (Ed.), International rvieu of research in mental retardation. Vol. 3 .
New York: Academic Press, 1968.

ZEAMAN, D., \& HOUSE, B. J. The role of attention in retardate discrimination learning. In N. R. Ellis (Ed.), Handbook of mental deficiency. New Yodk: McGraw-Hill, 1963 